\title{
Review Article \\ Optical Biosensors: A Revolution Towards Quantum Nanoscale Electronics Device Fabrication
}

\author{
D. Dey ${ }^{1}$ and T. Goswami² \\ ${ }^{1}$ Department of Engineering Physics, Tripura Institute of Technology, Narsingarh, Tripura-799009, India \\ ${ }^{2}$ Department of Electronics and Telecommunication, Tripura Institute of Technology, Narsingarh, Tripura-799009, India
}

Correspondence should be addressed to D. Dey, dh_dey@rediffmail.com

Received 16 June 2011; Accepted 9 August 2011

Academic Editor: Paul W. Doetsch

Copyright (C) 2011 D. Dey and T. Goswami. This is an open access article distributed under the Creative Commons Attribution License, which permits unrestricted use, distribution, and reproduction in any medium, provided the original work is properly cited.

The dimension of biomolecules is of few nanometers, so the biomolecular devices ought to be of that range so a better understanding about the performance of the electronic biomolecular devices can be obtained at nanoscale. Development of optical biomolecular device is a new move towards revolution of nano-bioelectronics. Optical biosensor is one of such nano-biomolecular devices that has a potential to pave a new dimension of research and device fabrication in the field of optical and biomedical fields. This paper is a very small report about optical biosensor and its development and importance in various fields.

\section{Introduction}

Optical biosensors are powerful alternative to conventional analytical techniques, for their particularly high specification, sensitivity, small size, and cost effectiveness $[1,2]$. Although promising developments of optical biosensors are being reported, but there are not many reports on applications of optical biosensor in practical field [3]. The research and technological development of optical biosensors have experienced an exponential growth during the last decade because this technology has a great potential for the direct, real-time and label-free detection of many chemical and biological substances [4]. Figure 1 shows the year on year development of biosensor market [5]. The success of the biosensor technology can be deduced for the increasing number of commercially available instruments. A highly multidisciplinary approach including microelectronics, MEMS, micro/nanotechnologies, molecular biology, nano-biotechnology, and chemistry are needed for the implementation of such new analytical devices [6-8]. Biosensing devices fabricated with optoelectronics micro/nanotechnologies are powerful devices which can fulfill these requirements. Optical biosensors offer great advantages over conventional analytical techniques. The selectivity of the biological sensing element offers the opportunity for development of highly specific devices for real-time analysis in complex mixtures, without the need for extensive sample pretreatment or large sample volumes. Optical biosensors are highly sensitive, rapid, reproducible, and simple-to-operate analytical tools. Despite optimism for the potential of biosensors, their emergence from the research laboratory to the marketplace has been slow. The obstacles to exploitation have been fundamentally related to the presence of biomaterial in the biosensor (immobilization of biomolecules on transducers, stability of enzymes, and antibodies), the development of the sensor device (sensitivity and reproducibility issues), and the integration of optical biosensors into complete systems. Another major problem for the realistic mass production of biosensors has been the cost factor. A biosensor system can be defined as the combination of different entities such as sampling, a biosensor, a system for replenishing information, and a data analysis system to implement a biological model which provides information to a human or automated controller. The integration of fluidics, electronics, separation technology, and biological subsystems is crucial for the development of biosensor systems. The sensor/sampling system biointerface is a key target for the construction of an integrated system. From the literature, there are many examples of biosensors successfully tested in a laboratory 


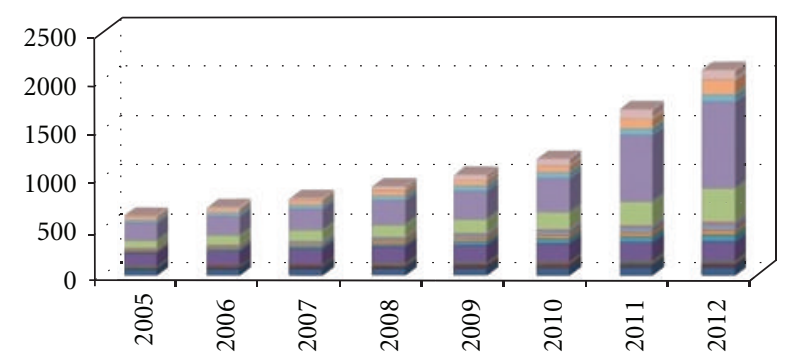

FIGURE 1: Graph showing the development of optical biosensor in the last decade.

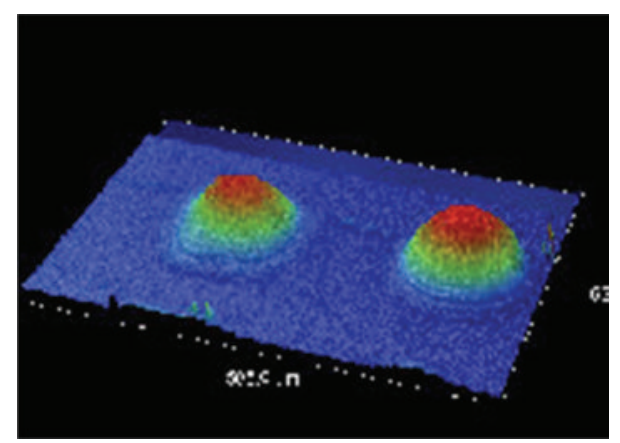

Figure 2: A typical optical biosensor.

or at prototype level but there are few examples, even in research, of integrated biosensor systems that offer automatic monitoring in complex matrices [9]. Figure 2 shows a basic prototype of an optical biosensor. The potential applications of biosensors in medical and biotechnological are numerous, and each has its own requirements in terms of the concentration of analyze to be measured, the required precision of output, the sample concentration required, the time taken to complete the probe, the time necessary for the biosensor to be ready to be used again, and the cleaning requirements of the system. The size of the possible market may also have an impact on the type of biosensor specified, as some are more amenable to mass production than others. In this review paper discussion is made on optical biosensor technology, bioreceptor systems, and methods of transduction. Optical biosensor research and development has been directed mainly towards health care, environmental applications, and biotech industry. The most commercially important application is the hand-held glucose meter used by diabetics. In cultivation field, no true biosensor systems have made an impact. The need for fast, online and accurate sensing opens up opportunities for biosensors in many different areas [10].

\section{Device Architecture and Detection Technique}

Depending on the method of signal transduction, biosensors can also be divided into different groups: electrochemical, optical, thermometric, piezoelectric, or magnetic. Optical biosensors are the most commonly reported class of biosensors. The detection typically relies on an enzyme system that catalytically converts analytes into products that can be oxidized or reduced at a working electrode, maintained at a specific potential. The main advantage of this optical transducer is the low cost and the use of biodegradable electrodes. An optical biosensor is a compact analytical device, having biological sensing element, integrated or connected to, an optical transducer system (Figure 2). The detection of specific binding of the analyte of interest to the complementary optical biorecognition element is immobilized on a suitable optical substrate [11]. The basic objective of optical biosensor is to produce an electronic signal which is proportional in magnitude or frequency corresponding to the concentration of a specific analyte or group of analytes, to which the biosensing element binds [12]. Optical biosensors can be classified by their biorecognition system. The main biological materials used in optical biosensor technology are the optocouple enzyme/substrate, antibody/antigen, and nucleic acids/complementary sequences. In addition, microorganisms, animal or plant whole cells and tissue slices can also be incorporated in the biosensing system. Recent advances and developments in the molecular optoelectronics offer an alternative approach involving the use of optical biometric recognition systems. Optical biosensors are increasingly impacting analytical technology for the detection of biological and chemical species. Figure 3 shows the architecture of an optical biosensor. Most of the integrated optical sensors make use of the evanescent field detection principle for sensing [13]. Deposition techniques such as screen printing and ink-jet printing allow printing of materials at very high precision and speed, producing large numbers of inexpensive and reproducible biosensors. Thin-film deposition techniques such as Langmuir-Blodgett technology [14] and Layer by Layer (LbL) technique [15] reported in some of my previous work are suitable for production of layers of few nanometer thicknesses which is important for the development of optical biosensors. Figure 4 represents the process of immobilization of biomolecules on solid substrate. Optical sensors are selective and sensitive devices for the detection of very low levels of chemicals and biological substances and for the measurement of molecular interactions in situ and in real time [16]. The advantages of the optical sensing are significantly improved when this approach is used in an integration scheme [17]. The technology of integrated optics allows the integration of several passive and active optical components onto the same substrate, allowing the flexible development of minimized compact sensing devices, with the additional possibility of fabrication of multiple sensors on one chip [18].

In recent times, a key stimulus for the development of optical biosensors has been the availability of highquality polymers and optoelectronic components. The optical biosensor format may involve direct detection of the analyte of interest or indirect detection through optically labeled probes, and the optical transducer may detect changes in the absorbance, luminescence, polarization, or refractive index $[19,20]$. The piezoelectric biosensor is based on measuring frequency changes directly related to mass change on the sensor surface. One of the main advantages of this technique is the real-time binding reaction detection, 


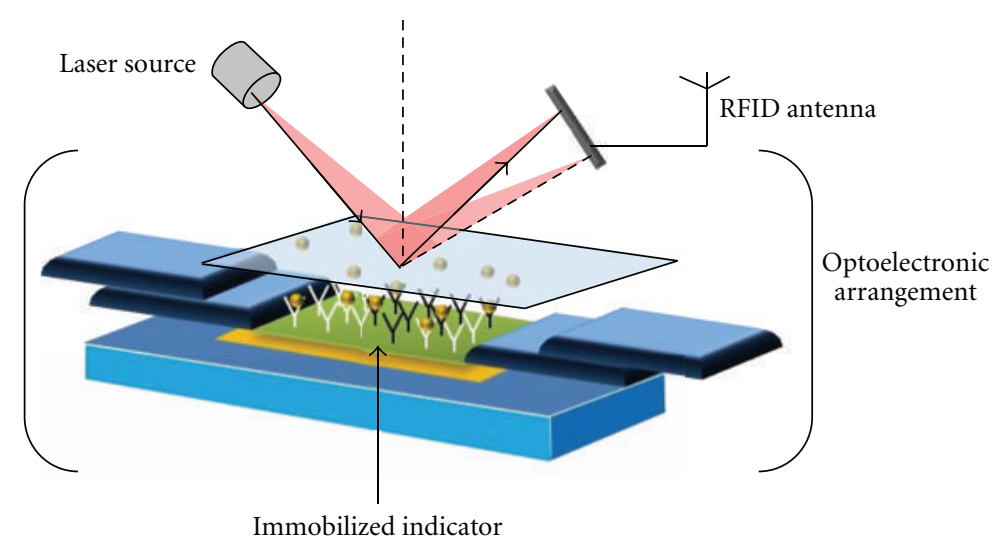

FIGURE 3: Architecture of an optical biosensor.

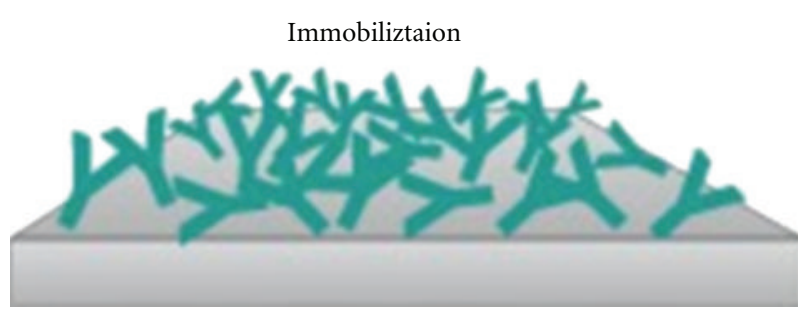

FIGURE 4: Immobilization of biomolecules.

allowing kinetic evaluation of affinity interactions (feature similar to the surface plasmon resonance biosensors) and, in addition, the low cost of the instrumentation required. Limitations of this transduction method are the need for a calibration of each crystal and the possible variability when coating the surface with the antigen or antibody [21].

There are two types of optical biosensors one based on surface resonance technology; almost all the commercially available optical biosensors depend on the surface morphology and therefore microelectronics technology allow the possibility for integration of optical, fluidics, and electrical function on one optical sensing circuit in order to obtain a complete lab-on-a-chip. But these types of optical biosensors have limitation of detection close to femtometer which is not achievable with this type of sensors in the unmodified format [22]. The other is optical nanomechanical biosensors. In this type of sensor microcantilever biosensors are a new class of high sensitivity biosensors able of performing local, high resolution, and label-free molecular recognition measurements [23]. Moreover, nanomechanical biosensors based on microcantilevers have been recently reported as a promisingly alternative to current DNA-chips allowing realtime monitoring of DNA without need of labeling. For that reason, we are working in the development of a portable multibiosensor microsystem based on an array of microcantilevers [24] able to detect analytes with femtomolar sensitivity and ability for discerning single base variations in DNA strands. The new trend to these nanomechanical biosensors is an excellent example of the application of micro- and nanotechnologies in the development of a new type of biosensors. Microcantilevers, such as those used in Atomic Force Microscopes, have been recently employed as this new class of biosensors [25]. The so-called nanomechanical biosensors have demonstrated that they are capable of detecting single-base mismatches in oligonucleotide hybridization without labeling as well as performing protein recognition [26] with extreme sensitivity. Among the advantages of nanomechanical biosensors are the potential for performing local, high resolution, and label-free molecular recognition measurements on a portable device. Also, the reduced sensor area allows drastic decrease of the reagent consumption. The working principle for nano mechanical optical biosensors relies on the induced surface stress that arises when molecules bind to a surface. When a monolayer of receptor molecules is immobilized on one side of the substrate then a cantilever deflection results from the differential surface stress between opposite sides of the cantilever. Molecular recognition produces also a change of the surface stress of the cantilever side with respect to the other side; laser beam reflects off the back of the cantilever to the position sensitive photodetector $[27,28]$.

\section{Molecular Level Interaction in Optical Biosensor}

The behavior of biomolecules adsorbed and immobilized on solid substrate during the fabrication of optical biosensors needs to be better understood for the specific interaction results in a change in one or more physicochemical properties $(\mathrm{pH}$ change, electron transfer, mass change, heat transfer, uptake or release of gases or specific ions) which are detected and may be measured by the transducer $[29,30]$. In recent years, there has been remarkable progress in surface morphology analysis using scanning electron microscopy and scanning probe microscope techniques such as atomic force microscopy (AFM) and scanning tunneling microscopy (STM) but unfortunately our knowledge of manipulation and improvement of protein stability is far from complete. The limited stability of proteins (enzymes, antibodies) acts as a brake on the development of biosensors. The development of DNA probes is a promising area of research in 
biosensors [31]. Deoxyribonucleic acid sensors (Figure 5) could be used to detect polymorphism or mutations in genes within plants, animals, and microorganisms. Biometric systems will also accelerate biosensor development and applications. Some researchers are trying to overcome the poor stability of biological molecules by developing artificial molecular recognition systems with predetermined selectivity for various substances. So far, molecular imprinted polymers have been prepared with high affinities for proteins, amino acid derivatives, sugars, vitamins, pesticides, and pharmaceuticals [32-35]. Some advantages of molecular imprinting versus biological receptors are the low cost, ease of synthesis, and relative long-term stability. The development of automated manufacturing technologies is extremely important in the commercial mass production of biosensors. Another area of intense research activity is fabrication of optical biosensor using silicon technology. In this process chips of nanosize are being developed. There are other techniques also such as microfabrication and micromachining technologies. It is now technically feasible to miniaturize and integrate complex components such as valves, mixers, and flow cells in a single chip along with the biosensor. Biosensor advancement in the commercial world could also be accelerated by the use of intelligent instrumentation, electronics, and multivariate signal-processing methods such as optometrics and artificial neural networks. Increasing attention will have to be paid to the engineering of both the basic components and the device as a whole. It is in this area where agricultural engineers will have a key role in applying their knowledge of systems to improve sampling, calibration, and data analysis to provide instructions for a farmer or processor rather than raw data. An optical biosensor that is adaptable to multiple photo analyses detection will allow spreading development costs over several products. These future improvements will produce devices more competitive with the presently available instruments and be able to operate under field conditions. One of the many applications of optical biosensor is the detection of E. coli and other bacteria and microparticles [36]. Optical biosensor is a quantum optical electrical phenomenon based on the fact that energy carried by photons of light can be transferred to electrons in a metal. The wavelength of light at which coupling occurs is characteristic of the particular metal and the environment of the metal surface illuminated. This transfer can be observed by measuring the amount of light reflected by the metal surface. The most common practical implementation of optical biosensor is to use a metal-coated optical prism, but other practical implementations have been demonstrated including metal-coated diffraction gratings, optical fibers, and planar waveguides. The SPR biosensor has potential for use in rapid, real-time detection and identification of bacteria and to study the interaction of organisms with different molecular species [37] described a piezoelectric biosensor for detection of Salmonella typhimurium. The device consists of a quartz crystal wafer sandwiched between two metal electrodes. These electrodes provide a means of connecting the device to an external oscillator circuit that drives the quartz crystal at its resonant frequency. A change in mass on the surface of the electrode thus changes

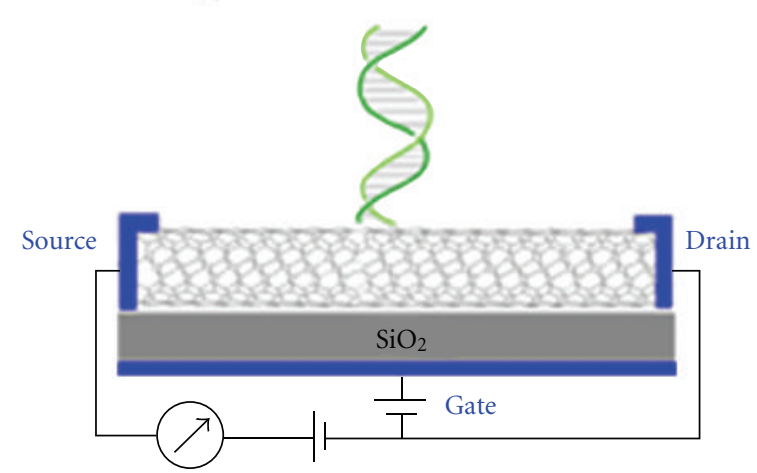

FIguRE 5: Diagram of DNA biosensor.

the resonant frequency of the quartz crystal microbalance (QCM) device. The antibody against Salmonella was immobilized onto the gold electrode-coated quartz crystal surface through a polyethylenimine deposition technique. Another biosensor direct method [38], in which Salmonella binding to specific antibodies attached to a waveguide surface were detected in minutes by measuring interferometrically the alteration in phase velocity of a guided optical wave. The biosensor was able to detect S. typhimurium in chicken. In an optical biosensor the light travels inside the waveguide, confined within the structure by the method of Total Internal Reflection (TIR). The light is transmitted through a model of the electromagnetic field called "guided modes" [39] and light is confined inside those modes; there is a part of EM wave that travels through a region that extends outward, around a hundred of nanometers, into the medium surrounding the waveguide. This EW field can be used for sensing purposes. When a receptor layer is immobilized onto the waveguide, exposure of such a surface to the partner analyte molecules produces a (bio)chemical interaction, which takes place into the surface of the waveguide and induces a change in its optical properties that is detected by the evanescent wave. The extent of the optical change will depend on the concentration of the analyte and on the affinity constant of the interaction, obtaining, in this way, a quantitative sensor of such interaction. Figure 6 shows the phenomenon of interaction between light waves and biomolecules. Application and commercialization of biosensor technology has lagged behind the output of research laboratories. Although many biosensor-related patents are being registered every year, very few play a prominent role in clinical diagnostic, food industry, environmental and biomedical uses $[40,41]$. For this slow technology transfer from the research laboratories to the marketplace: cost considerations, stability and sensitivity issues, quality assurance, and instrumentation design [42]. Many of the main barriers are technical, methods of sensor calibration, methods of producing inexpensive and reliable sensors, stabilization and storage of biosensors, and total integration of the sensor system [43]. 


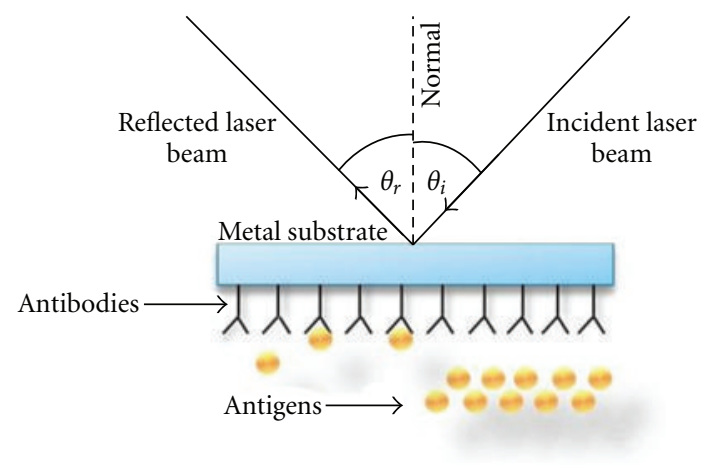

FIgURE 6: Interaction of biomolecules with light.

\section{Recent Developments and Future Outlook}

The advantages of optical biosensors are their speed, the immunity of the signal to electrical or magnetic interference, and the potential for higher information content (spectrum of information available) but the main drawback can be the high cost of some instrumentation. Optical biosensor in the last decade has developed to a great extent in every field but there is still a long way to replace completely the conventional methods of the optical biosensor technology in many fields and, specially, in biomedical field. To achieve such objective, we still need to develop optical biosensors able to detect, in a direct way at very low levels (picomolar to femtomolar) of a great number of substances in the areas of environmental monitoring, industrial and food process, health care, biomedical technology, clinical analysis, and so forth $[44,45]$. For that reason, there is an increasing interest in systems based on micro/nanotechnologies for the development of ultrasensitive and miniaturized biosensors [46] which can be easily integrated in microsystems. There are various technical difficulties for fabrication of optical biosensor but still more research efforts are needed for finding out better alternatives. Some of them are as follows.

(1) Immobilization. Immobilization of biomaterials is the hardest difficulty in the process of fabrication of optical biosensor. Material losses are observed during the process of immobilization of biomolecules on solid substrate. In some reports prominent material loss is observed [47]. Some new methods are being also reported to minimize the material loss [48] which can be useful for further development and research in the field of biosensors.

(2) Contamination. There is a big problem of biomolecules and chemicals used in the biosensors leaking out of the biosensor which may create contamination. To avoid contam-ination, biomolecules should be attached to the transducer as strongly as possible. But the behavior of biomolecules when absorbed on the surface is not well understood clearly till now.

(3) Uniformity. Optical biosensors that are fabricated by different processes can be easily reproduced if they are uniform.
This immobilization can be made by using LB technique or by spin-coating technique where the molecular uniformity is obtained with highest degree of precision $[49,50]$.

(4) Selection Range. The selection range should be large in order to detect alot of variety of biomaterials.

(5) Sterilization. Sensor's biomolecules may get destroyed if nonsterile probes are used.

(6) Expensive. Low-cost optical biosensors have to be developed for mass use and to have a better equipment to fight out different medical and technological problems.

At present, with the threat of bioterrorism, the development of faster, reliable, accurate, portable, and low-cost biosensors has become more important.

\section{Conclusion}

The application of biotechnology would seem to be vital in maintaining the productivity and health of crops and livestock in the face of environmental concerns, limited resources, and population increases. Biosensors could play an important role in providing powerful analytical tools to the agricultural diagnosis sector, particularly where rapid, lowcost, high-sensitivity, and specificity measurements in field situations are required. This review summarised ongoing developments in this field. A range of molecules with biorecognition properties can be used as the sensing element in biosensors. A wide range of transducers is also available to engineer new biosensing devices. There are many different ways to combine biology, chemistry, physics, mathematics, and engineering in order to develop new biosensors with applications in agriculture. The promise has been shown by new optical biosensor technology that it is very real, however there are some technological obstacles that need to be overcome. Advances in areas such as surface chemical analysis, protein stabilization, automated manufacturing technologies would widen the market and allow biosensors to be more competitive in the agricultural market. In this paper we have discussed various biosensors in detail. The survey initially introduces the basic concepts of optical biosensor. Overview of different types of optical biosensors is also given along with working principles, constructions, advantages, and applications of many biosensors are presented. The paper also points out the advances of optical biosensors during the last two decades. We have presented the development of different micro- and nano-biosensor platforms in which we integrate the bio, nano, micro and other aspects in order to achieve highly sensitive and miniaturized devices.

\section{References}

[1] X. Luo, J. Xu, W. Zhao, and H. Chen, "Glucose biosensor based on ENFET doped with $\mathrm{SiO} 2$ nanoparticles," Sensors and Actuators: B, vol. 97, no. 2-3, pp. 249-255, 2004.

[2] W. Sant, M. Pourciel, J. Launay, T. Do Conto, A. Martinez, and P. Temple-Boyer, "Development of chemical field effect 
transistors for the detection of urea," Sensors and Actuators: B, vol. 95, no. 1-3, pp. 309-314, 2003.

[3] J. Chen, J. Chou, T. Sun, and S. Hsiung, "Portable urea biosensor based on the extended-gate field effect transistor," Sensors and Actuators: B, vol. 91, no. 1-3, pp. 180-186, 2003.

[4] A. P. Soldatkin, J. Montoriol, W. Sant, C. Martelet, and N. Jaffrezic-Renault, "A novel urea sensitive biosensor with extended dynamic range based on recombinant urease and ISFETs," Biosensors and Bioelectronics, vol. 19, no. 2, pp. 131135, 2003.

[5] http://www.sensorsmag.com/list/specialty-markets/medical16.

[6] R. P. H. Kooyman and L. M. Lechuga, "Handbook of Biosensors: Medicine," in Food and the Environment, E. Kress-Rogers, Ed., p. 169, CRC Press, Boca Raton, Fla, USA, 1997.

[7] L. M. Lechuga, F. Prieto, and B. Sepúlveda, "Interferometric biosensors for environmental pollution detection," in Optical Sensors for Industrial, R. Narayanaswamy and O. S. Wolfbeis, Eds., Springer, New York, NY, USA, 2003.

[8] R. G. Freeman, K. C. Grabar, K. J. Allison et al., "Selfassembled metal colloid monolayers: an approach to SERS substrates," Science, vol. 267, no. 5204, pp. 1629-1632, 1995.

[9] G. Chumanov and S. Malynych, "Coupled planar silver nanoparticle arrays as refractive index sensors," Journal of Optics A: Pure and Applied Optics, vol. 8, no. 4, pp. S144-S147, 2006.

[10] S. Y. Chou and P. R. Krauss, "Imprint lithography with sub$10 \mathrm{~nm}$ feature size and high throughput," Microelectronic Engineering, vol. 35, no. 1-4, pp. 237-240, 1997.

[11] M. Li, L. Chen, W. Zhang, and S. Y. Chou, "Pattern transfer fidelity of nanoimprint lithography on six-inch wafers," Nanotechnology, vol. 14, no. 1, pp. 33-36, 2003.

[12] D. C. Turner, C. Y. Chang, K. Fang, S. L. Brandow, and D. B. Murphy, "Selective adhesion of functional microtubules to patterned silane surfaces," Biophysical Journal, vol. 69, no. 6, pp. 2782-2789, 1995.

[13] A. D. McFarland and R. P. van Duyne, "Single silver nanoparticles as real-time optical sensors with zeptomole sensitivity," Nano Letters, vol. 3, no. 8, pp. 1057-1062, 2003.

[14] S. Biswas, S. A. Hussain, and D. Bhattacharjee, "Spectroscopic characterizations of nonamphiphilic 2,5-bis (5-Tert-ButylBenzoxazolyl)-thiophene molecules at the air-water interface and in langmuirblodgett films," Surface Review and Letters, vol. 15 , no. 6 , pp. $1-8,2008$.

[15] D. Dey, S. A. Hussain, R. K. Nath, and D. Bhattacharjee, "Preparation and characterization of an anionic dyepolycation molecular films by electrostatic layer-by-layer adsorption process," Spectrochimica Acta: Part A, vol. 70, no. 2, pp. 307-312, 2008.

[16] N. Nath and A. Chilkoti, "A colorimetric gold nanoparticle sensor to interrogate biomolecular interactions in real time on a surface," Analytical Chemistry, vol. 74, no. 3, pp. 504-509, 2002.

[17] P. Schuck, "Use of surface plasmon resonance to probe the equilibrium and dynamic aspects of interactions between biological macromolecules," Annual Review of Biophysics and Biomolecular Structure, vol. 26, pp. 541-566, 1997.

[18] W. Knoll, "Interfaces and thin films as seen by bound electromagnetic waves," Annual Review of Physical Chemistry, vol. 49, no. 1, pp. 569-638, 1998.

[19] R. Sosnowski, M. J. Heller, E. Tu, A. H. Forster, and R. Radtkey, "Active microelectronic array system for DNA hybridization, genotyping and pharmacogenomic applications," Psychiatric Genetics, vol. 12, no. 4, pp. 181-192, 2002.
[20] M. Minunni, S. Tombelli, M. Mascini, A. Bilia, M. C. Bergonzi, and F. F. Vincieri, "An optical DNA-based biosensor for the analysis of bioactive constituents with application in drug and herbal drug screening," Talanta, vol. 65, no. 2, pp. 578-585, 2005.

[21] J. F. Rusling, E. G. Hvastkovs, D. O. Hull, and J. B. Schenkman, "Biochemical applications of ultrathin films of enzymes, polyions and DNA," Chemical Communications, no. 2, pp. 141-154, 2008.

[22] M. Keusgen, "Biosensors: new approaches in drug discovery," Nature, vol. 89, no. 10, pp. 433-444, 2002.

[23] P. Vattanaviboon, K. Sangseekhiow, P. Winichagoon, and C. Promptmas, "Detection and haplotype differentiation of Southeast Asian $\alpha$-thalassemia using polymerase chain reaction and a piezoelectric biosensor immobilized with a single oligonucleotide probe," Translational Research, vol. 151, no. 5, pp. 246-254, 2008.

[24] H. Shi, T. Xia, A. E. Nel, and J. I. Yeh, "Part II: coordinated biosensors-development of enhanced nanobiosensors for biological and medical applications," Nanomedicine, vol. 2, no. 5, pp. 599-614, 2007.

[25] R. Gambari and G. Feriotto, "Surface plasmon resonance for detection of genetically modified organisms in the food supply," Journal of AOAC International, vol. 89, no. 3, pp. 893897, 2006.

[26] A. Logrieco, D. W. Arrigan, K. Brengel-Pesce, P. Siciliano, and I. Tothill, "DNA arrays, electronic noses and tongues, biosensors and receptors for rapid detection of toxigenic fungi and mycotoxins: a review," Food Additives and Contaminants, vol. 22, no. 4, pp. 335-344, 2005.

[27] E. R. Richter, "Biosensors: applications for dairy food industry," Journal of Dairy Science, vol. 76, no. 10, pp. 3114-3117, 1993.

[28] D. Ivnitski, D. J. O’Neil, A. Gattuso, R. Schlicht, M. Calidonna, and R. Fisher, "Nucleic acid approaches for detection and identification of biological warfare and infectious disease agents," BioTechniques, vol. 35, no. 4, pp. 862-869, 2003.

[29] I. Palchetti and M. Mascini, "Nucleic acid biosensors for environmental pollution monitoring," Analyst, vol. 133, no. 7, pp. 846-854, 2008.

[30] F. Dondero, L. Piacentini, F. Marsano et al., "Gene transcription profiling in pollutant exposed mussels (Mytilus spp.) using a new low-density oligonucleotide microarray," Gene, vol. 376, no. 1-2, pp. 24-36, 2006.

[31] P. Venier, C. de Pittà, A. Pallavicini et al., "Development of mussel mRNA profiling: can gene expression trends reveal coastal water pollution?" Mutation Research, vol. 602, no. 1-2, pp. 121-134, 2006.

[32] M. J. LaGier, J. W. Fell, and K. D. Goodwin, "Electrochemical detection of harmful algae and other microbial contaminants in coastal waters using hand-held biosensors," Marine Pollution Bulletin, vol. 54, no. 6, pp. 757-770, 2007.

[33] A. Kahvejian, J. Quackenbush, and J. F. Thompson, "What would you do if you could sequence everything?" Nature Biotechnology, vol. 26, no. 10, pp. 1125-1133, 2008.

[34] J. Shendure and H. Ji, "Next-generation DNA sequencing," Nature Biotechnology, vol. 26, no. 10, pp. 1135-1145, 2008.

[35] S. Nagl, M. Schaeferling, and O. S. Wolfbeis, "Fluorescence analysis in microarray technology," Microchimica Acta, vol. 151, no. 1-2, pp. 1-21, 2005.

[36] H. Sirringhaus, P. J. Brown, R. H. Friend et al., "Twodimensional charge transport in self-organized, high-mobility conjugated polymers," Nature, vol. 401, no. 6754, pp. 685-688, 1999. 
[37] J. Kong, N. R. Franklin, C. Zhou et al., "Nanotube molecular wires as chemical sensors," Science, vol. 287, no. 5453, pp. 622625, 2000.

[38] M. Schäferling and S. Nagl, "Optical technologies for the read out and quality control of DNA and protein microarrays," Analytical and Bioanalytical Chemistry, vol. 385, no. 3, pp. 500-517, 2006.

[39] N. Jaffrezic-Renault, C. Martelet, P. Clechet et al., "Comparison of two transduction modes for design of microbiosensors applicable to detection of pesticides (S\&M 0233)," Sensors and Materials, vol. 8, pp. 161-168, 1996.

[40] M. Dufva, "Fabrication of high quality microarrays," Biomolecular Engineering, vol. 22, no. 5-6, pp. 173-184, 2005.

[41] A. Sassolas, B. D. Leca-Bouvier, and L. J. Blum, "DNA biosensors and microarrays," Chemical Reviews, vol. 108, no. 1, pp. 109-139, 2008.

[42] S. Campanaro, C. Romualdi, M. Fanin et al., "Gene expression profiling in dysferlinopathies using a dedicated muscle microarray," Human Molecular Genetics, vol. 11, no. 26, pp. 3283-3298, 2002.

[43] M. Campàs and I. Katakis, "DNA biochip arraying, detection and amplification strategies," Trends in Analytical Chemistry, vol. 23, no. 1, pp. 49-62, 2004.

[44] D. Branton, D. W. Deamer, A. Marziali et al., "The potential and challenges of nanopore sequencing," Nature Biotechnology, vol. 26, no. 10, pp. 1146-1153, 2008.

[45] J. A. Shendure, G. J. Porreca, and G. M. Church, "Overview of DNA sequencing strategies," Current Protocols in Molecular Biology, no. 81, pp. 7.1.1-7.1.11, 2008.

[46] J. Eid, A. Fehr, J. Gray et al., "Real-time DNA sequencing from single polymerase molecules," Science, vol. 323, no. 5910, pp. 133-138, 2009.

[47] S. A. Hussain, D. Dey, D. Bhattacharjee, and S. Sinha, "Immobilization of single strand DNA on solid substrate," Chemical Physics Letters, vol. 450, no. 1-3, pp. 49-54, 2007.

[48] D. Dey, S. A. Hussain, and D. Bhattacharjee, "Layer-by-layer self assembled films of rosebengal," International Journal of Modern Physics B. In press.

[49] S. A. Hussain, M. N. Islam, H. Leeman, and D. Bhattacharjee, "Aggregation of p-terphenyl along with PMMA/SA at the Langmuir and Langmuir-Blodgett films," Surface Review and Letters, vol. 15, no. 4, pp. 1-9, 2008.

[50] D. Dey, M. N. Islam, S. A. Hussain, and D. Bhattacharjee, "Effect of temperature and ionic concentration on selfassembled films of Chicago sky blue," Chinese Physics Letters, vol. 25, no. 10, pp. 3732-3734, 2008. 

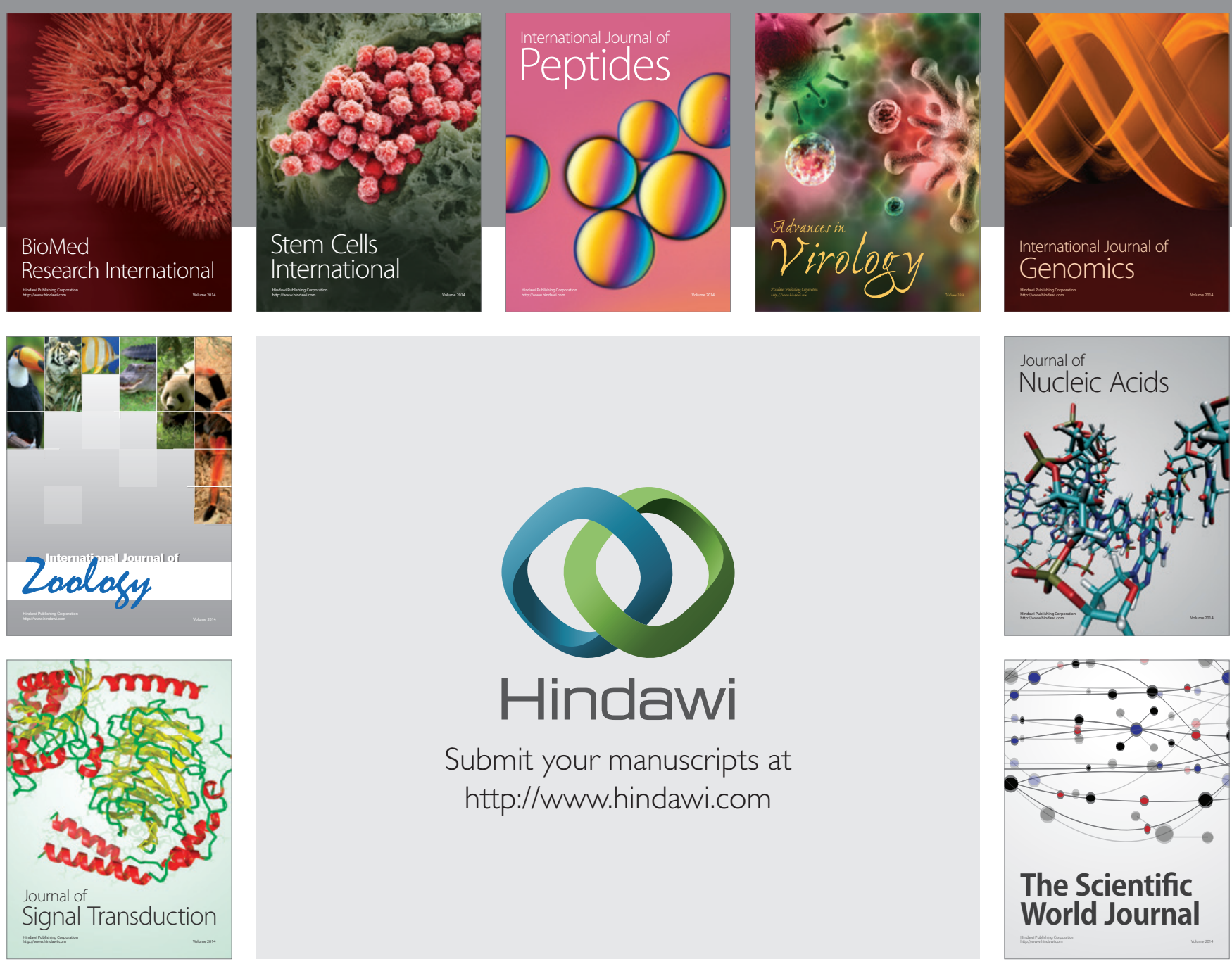

Submit your manuscripts at

http://www.hindawi.com
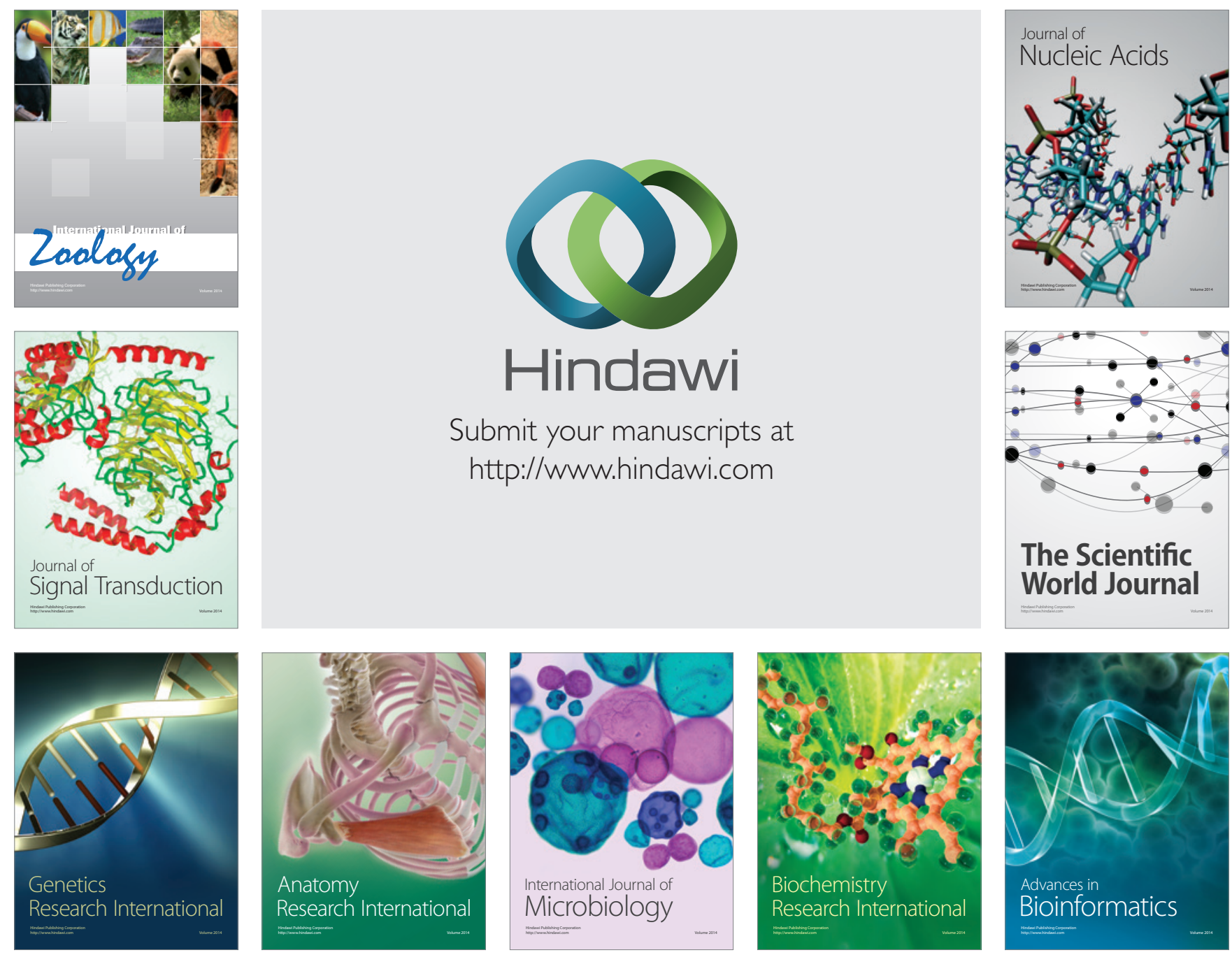

The Scientific World Journal
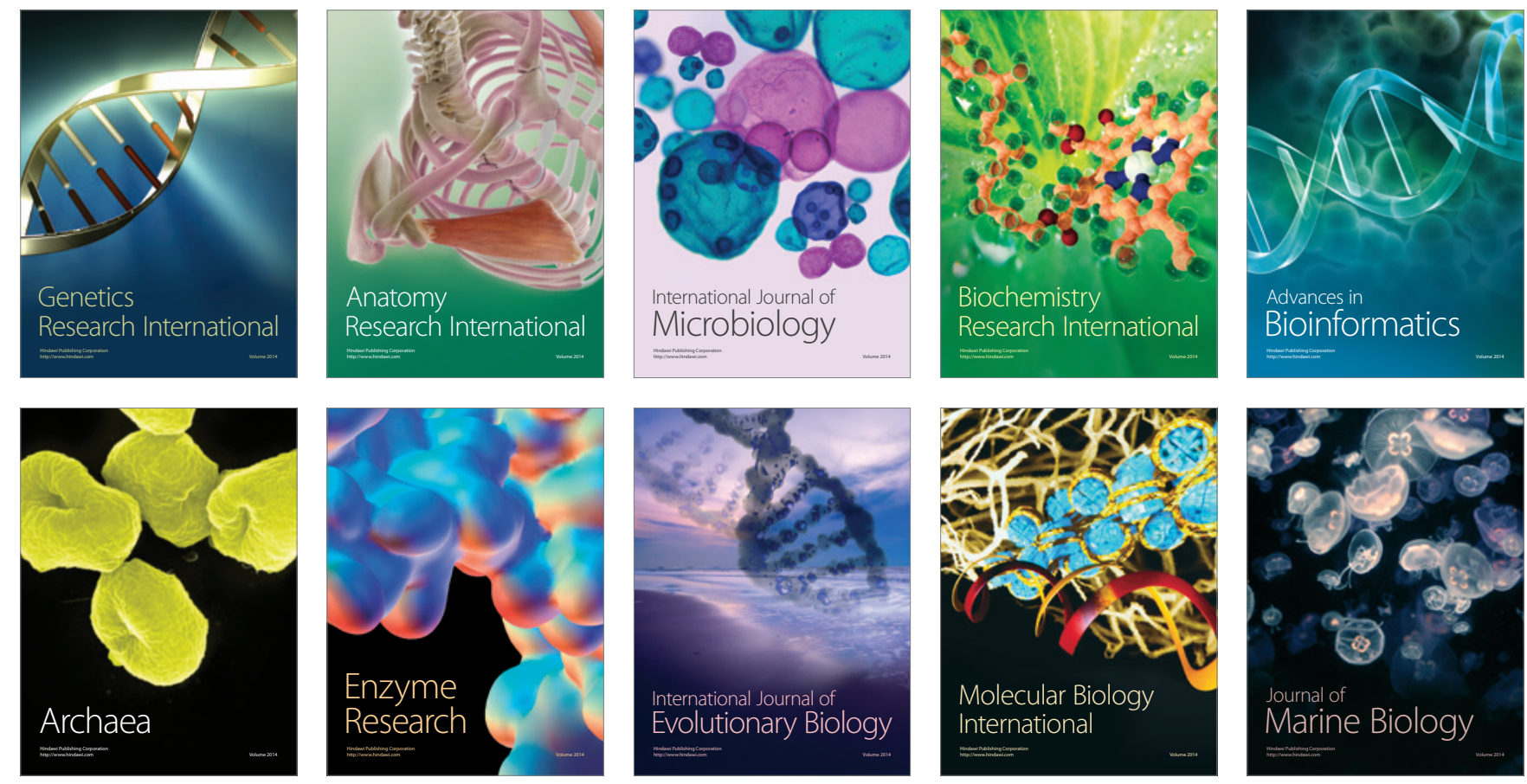